\title{
A Wideband 77GHz, 17.5dBm Power Amplifier in Silicon
}

\author{
Abbas Komijani and Ali Hajimiri \\ Department of Electrical Engineering \\ California Institute of Technology, Pasadena, CA 91125, USA
}

\begin{abstract}
A 77GHz, $+17.5 \mathrm{dBm}$ fully-integrated power amplifier (PA) with $50 \Omega$ input and output matching is fabricated in a $0.12 \mu \mathrm{m}$ SiGe BiCMOS process. The power amplifier achieves a peak power gain of $17 \mathrm{~dB}$ and a maximum single-ended output power of $+17.5 \mathrm{dBm}$ with $12.8 \%$ of power-added efficiency (PAE). It has a 3dB bandwidth of $15 \mathrm{GHz}$ and draws $165 \mathrm{~mA}$ from a $1.8 \mathrm{~V}$ supply. Microstrip tubs are used as the transmission line structure resulting in large isolation between adjacent lines, enabling integration of the PA in a small area of $0.6 \mathrm{~mm}^{2}$.
\end{abstract}

\section{INTRODUCTION}

Higher frequency millimeter ( $\mathrm{mm}$ ) wave bands offer exciting opportunities for various applications such as short-range communication (e.g., 60GHz band) and automotive radar (e.g., $77 \mathrm{GHz}$ band) [1][2][3]. Penetration of silicon integrated circuits into these bands can bring the unchallenged reign of compound semiconductors at these frequencies to an end. The unmatched levels of integration offered by silicon integrated circuits will enable a new level of integration and novel architectures encompassing microwave, analog, and digital blocks [4][5].

Perhaps the most challenging building block at mm-wave frequencies is the power amplifier (PA). The continued scaling of the transistor dimensions results in an inevitable reduction in the breakdown voltages that in turn aggravates the PA design challenges and necessitates novel design techniques at high frequencies. On the other hand, the mm-wave frequencies facilitate the use of on-chip transmission lines for power combing and impedance matching while providing high levels of on-chip isolation between various blocks. Such liberal use of transmission lines at lower frequencies is prohibited by their length (and hence loss). These concepts are demonstrated in this paper through the design of a fully integrated $77 \mathrm{GHz}$ power amplifier in silicon.

\section{FREQUENCY BAND}

In a collision-avoidance automotive radar, in order to resolve cars that are closely spaced at a far away distance, a large antenna directivity is required. For example two cars in adjacent lanes ( 3 meters apart) that are 100 meters away, have an angular separation of just $1.7^{\circ}$. In a typical short and long-range vehicular radar a directivity of $>20 \mathrm{~dB}$ and $>36 \mathrm{~dB}$ is required, respectively [6]. With a typical $\sim 8 \mathrm{~dB}$ directivity of a patch antenna, approximately $9 \times 4$ and $33 \times 31$ array elements are needed for the short-range and long-range, respectively. This

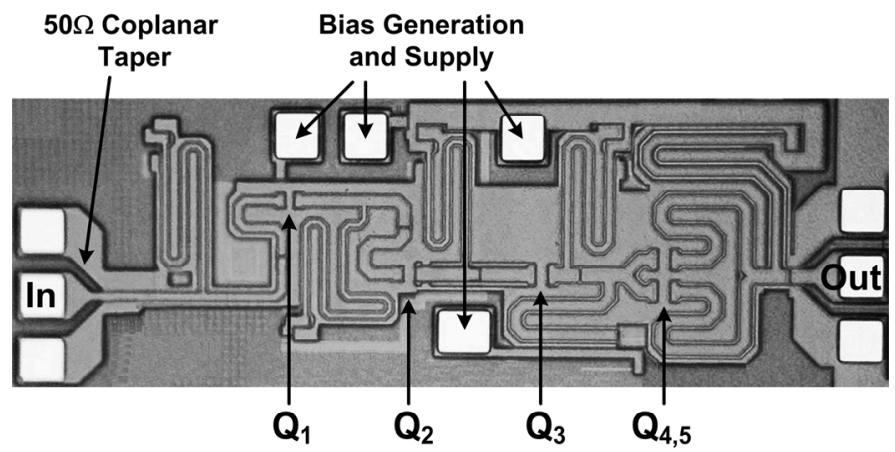

Fig. 1. Die micrograph of the $77 \mathrm{GHz}$ power amplifier, chip size: $1.35 \times 0.45$ $\mathrm{mm}^{2}$.

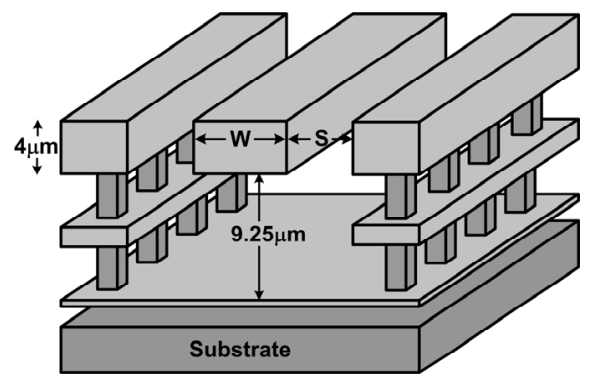

Fig. 2. Microstrip tub transmission line structure used for matching in the amplifier.

explains the need to operate at very high frequencies, such as 76$77 \mathrm{GHz}$ band, to be able to reduce the system size for such large number of elements.

\section{AMPLIFIER DESIGN}

The power amplifier has been designed in a $0.12 \mu \mathrm{m}$ BiCMOS process featuring $\mathrm{SiGe}$ transistors with a cutoff frequency of $f_{T} \approx 200 \mathrm{GHz}$ [7]. Die photo of the amplifier is shown in Fig. 1. The back-end consists of 5 metal layers with three copper layers and a thick $4 \mu \mathrm{m}$ aluminum layer as top metal for low-loss interconnects. The breakdown voltages of the transistors are $B V_{C E O} \approx 1.7 \mathrm{~V}$ and $B V_{C B O} \approx 5.5 \mathrm{~V}$. Substrate resistivity is $\sim 14$ $\Omega . c m$.

\section{A. Microstrip Tub as the Transmission Line Structure}

The microstrip tub structure shown in Fig. 2 is used throughout the amplifier for matching. The presence of the side shields increases isolation between adjacent lines, allowing a compact layout. Fig. 3 shows the isolation between two adjacent $50 \Omega$ lines vs. their center-to-center spacing. The lines are 


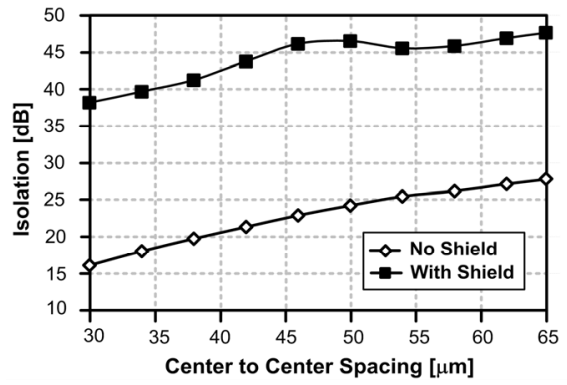

Fig. 3. The simulated isolation between two side-by-side $400 \mu \mathrm{m}, 50 \Omega$ microstrip lines with shield $(\mathrm{W}=5 \mu \mathrm{m}, \mathrm{S}=7.5 \mu \mathrm{m})$ and without shield $(\mathrm{W}=$ $13 \mu \mathrm{m})$.

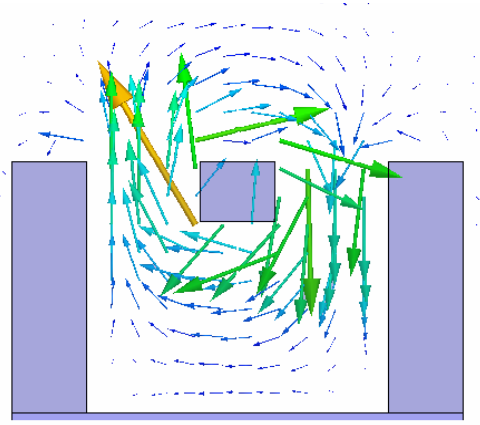

Fig. 4. The magnetic field distribution of the microstrip tub structure, showing most of the return current coming from the side shields.

implemented using the top three metals of the process. The lower two metals are left for routing of low frequency signals. The use of side shield increases isolation by more than $20 \mathrm{~dB}$. The transmission lines used in the amplifier are simulated using IE3D and their loss at $77 \mathrm{GHz}$ is less than $1.5 \mathrm{~dB} / \mathrm{mm}$.

Fig. 4. shows the magnetic field distribution in the transmission line, simulated with a 3D field solver (Ansoft HFSS). Each arrow indicates the direction and magnitude of the magnetic field at the base of the arrow. The tangential component of the magnetic fields is a clear indication of the surface current in metals. As can be seen from the figure, the bottom plate carries very little current (small tangential component of the magnetic field) while the shield carries most of the return current. In this respect the structure behavior is more similar to a coplanar structure, with bottom metal isolating it from substrate. The tub shape also reduces surface wave propagation, improving isolation between elements and hence simplifying single-chip system integration. Since the PA is the highest-power block in a transceiver, it is critical to minimize the interference generated by PA to sensitive elements in the transceiver (such as VCO).

\section{B. Circuit Architecture}

The schematic of the amplifier is shown in Fig. 5. The amplifier consists of 4 gain stages, where output stage is designed for maximum efficiency and the other stages are designed for maximum gain. The last three stages use 1, 2, and 4 identical transistor cells. This geometric increase in transistor size from stage to stage ensures that the output transistors will enter compression first as long as the preceding stages have at least $3 \mathrm{~dB}$ of gain. Carefully designed CPW tapers absorb pad parasitics, providing a $50 \Omega$ impedance at the input and output microstrip ports of the PA. All the transistors have single emitter stripe, use minimum emitter width of $0.12 \mu \mathrm{m}$, and have two base

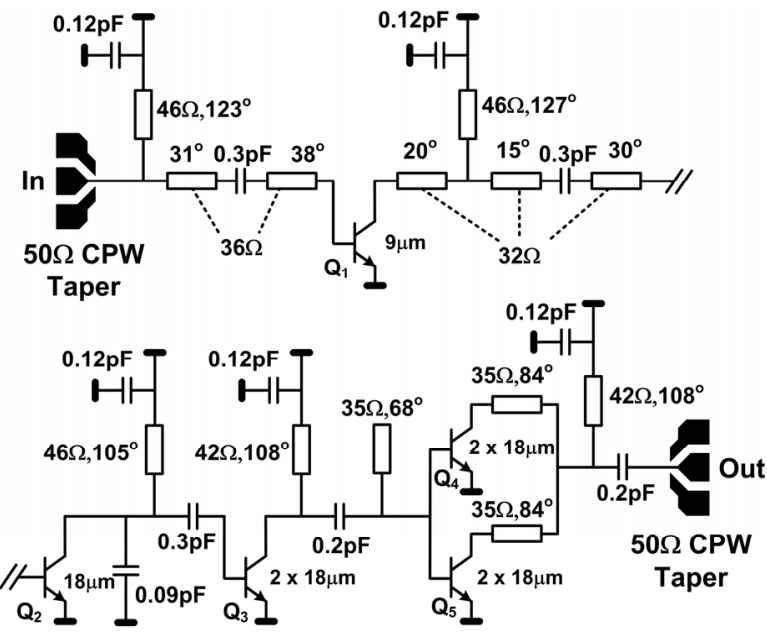

Fig. 5. Schematic of the $77 \mathrm{GHz}$ power amplifier including element values.

and collector contacts (CBEBC configuration). For reliable operation, the collector junction has more than the minimum number of contacts possible (three rows of long rectangular vias in parallel). The amplifier is biased in class-AB mode. Transistors are biased at their maximum $f_{\max }(1.2 \mathrm{~mA}$ for $1 \mu \mathrm{m}$ of emitter length).

When the power amplifier is driven into saturation, the collector voltage of output transistor can go up to more than twice the supply voltage. A low open-base collector-emitter breakdown voltage, $B V_{C E O}$, of $1.7 \mathrm{~V}$ limits the possible supply voltage to about $0.9 \mathrm{~V}$ for a large drive impedance in the base.

In a normal silicon transistor, maximum dielectric breakdown field and velocity saturation pose a rather fundamental breakdown voltage vs. speed limitation [8][9]. But in the process used the $B V_{C E O}$ limit is set by the impact ionization effect, in which the electron-hole pairs generated by accelerated electrons constitute base current. If the base is driven with lower source impedance this breakdown voltage will increase. In this case, voltage swing is limited by $B V_{C E R}$ rather than $B V_{C E O}$, where in the process used $B V_{C E R}$ is around $4 \mathrm{~V}$ for $R_{B}$ equal to $300 \Omega$ [3]. Therefore, the bias circuitry is designed to provide a base resistance of $300 \Omega$ for the transistors in the amplifier.

\section{Design of the Matching Networks}

The matching networks use series transmission lines and parallel shorted-stubs for power match between different stages. At input of the last stage an open stub provides lower matching network loss. At the output of second stage this was achieved with a parallel MIM capacitor.

The capacitors at the end of shorted parallel stubs are in parallel with a series RC network (which is not shown in Fig. 5 for simplicity). Careful choice of $\mathrm{R}$ and $\mathrm{C}$ reduces gain of the amplifier at low frequencies, enhancing stability.

The optimum impedance at the collector of each stage is determined with a large-signal power match. Similar to [10], a load pull simulation is performed to find the best load for the transistor. For the output stage this point is chosen to maximize the efficiency and for the other stages to maximize gain. Fig. 6 shows the result of the load pull simulations for all the four 

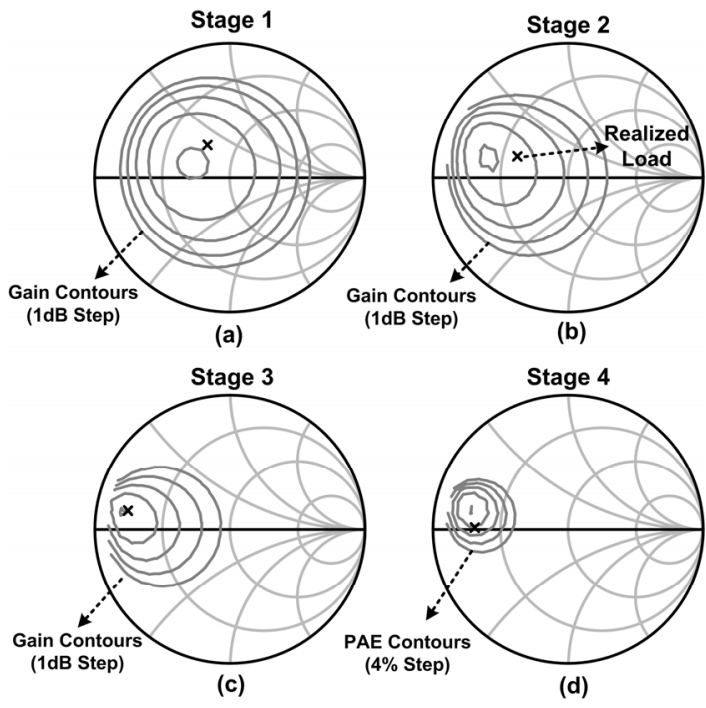

Fig. 6. Load pull simulation of the four stages of the power amplifier, together with the actual realized load impedances.

stages. These gain and PAE contours have steps of $1 \mathrm{~dB}$ and $4 \%$, respectively. The contours become denser as we move toward the output stage, indicating larger sensitivity of the amplifier to matching errors.

The realized impedance is not exactly at the center of the contours. This is more obvious in the case of output stage where the realized load provides a PAE that is $4 \%$ lower than maximum possible PAE. This is because the optimum load impedance is not the only criterion in the design of the matching network. Loss of the matching network also needs to be minimized during the design of matching network. A weighted least-mean-square optimization with gradient-descent scheme was used to choose the length and characteristic impedance of the matching networks. The optimization goal is to minimize the sum of the squares of the distance to the optimum load point and the loss in the matching network simultaneously. Therefore, for having a reasonable passive efficiency, the realized load is not exactly at the center of load pull contours. Due to a higher sensitivity of the output stage to mismatch, a slightly different design criterion was chosen for the output stage. Consequently, the output stage has the largest passive loss (2.2dB).

\section{Simulation and Layout Methodology}

The circuit was simulated in ADS. Electromagnetic simulations based on method of moments were performed using IE3D to design coplanar tapers and verify transmission line models and non-idealities like bends and T-junctions.

Parasitic capacitors are extracted on local nodes where the capacitance is not part of the distributed transmission-line structure. These nodes include connections to transistors, where top-level signal should travel to the lower levels closer to substrate. Parasitic collector-base capacitance is very important as it will be multiplied due to the Miller effect and appear at input or can even cause oscillation. Careful layout techniques minimized the overlap of the collector and base connections and reduced this parasitic capacitor to less than $1 \mathrm{fF}$ for all the transistors.

\section{E. Wideband Design}

Although the parasitic layout capacitances can be absorbed into the matching networks, they reduce bandwidth of the amplifier. One way to explain this behavior is by the Bode-Fano criterion [11]. If a lossless network is used to match a parallel RC load, there is an upper limit on the broadband match that can be achieved. More specifically:

$$
\int_{0}^{\infty} \ln \frac{1}{|\Gamma(\omega)|} d \omega \leq \frac{\pi}{R C}
$$

where $\Gamma(\omega)$ is the reflection coefficient seen looking into the arbitrary lossless matching network. Therefore by increasing the load capacitance the quality of the match will degrade, i.e., either the reflection coefficient must increase or the bandwidth has to be reduced. Though it's possible to have a wideband and flat gain by introducing large mismatch [11], load pull simulations in Fig. 6 show that the resulting mismatch will decrease gain and PAE of the amplifier, significantly, particularly at the output stage.

We minimized parasitic capacitances by a vertical wedge-type via array. The largest ratio of parasitic capacitance to device capacitance $(60 \%)$ occurs at the output of the first stage, with $16.5 \mathrm{fF}$ parasitic capacitance.

\section{Measurement Setup}

The small-signal gain of the amplifier was measured with HP $8757 \mathrm{E}$ scalar network analyzer. The network analyzer sweeps the output frequency of a high-power W-band back-wave oscillator (BWO) from Resonance Instruments Inc. This is done with a 705B millimeter wave sweeper from Micro-Now Instrument Co. The signal is fed through a WR-10 waveguide to a Pico-Probe WR-10 GSG probe. To calibrate the network analyzer first a thru measurement was done and then the PA was inserted in place of the thru.

The BWO has a fixed output power, which changes with frequency. To measure large-signal parameters of the amplifier, a variable attenuator (Millitech DRA-10-R000) with Agilent W8486A W-band power sensor was used. The loss of the probe was measured and de-embedded.

\section{Measurement Results}

The simulated and measured small-signal gain of the amplifier is shown in Fig. 7. The amplifier has a peak gain of $17 \mathrm{~dB}$ around $75 \mathrm{GHz}$. Normally the W-band waveguide measurement setup is used for $75-110 \mathrm{GHz}$ band, but the TE10 mode cutoff frequency for this waveguide is $59 \mathrm{GHz}$ and will not affect the measurement results between $65-75 \mathrm{GHz}$ significantly. The amplifier has a $3 \mathrm{~dB}$ bandwidth of at least $15 \mathrm{GHz}$ and has gain up to $94 \mathrm{GHz}$. A good match between simulated and measured results is observed. 


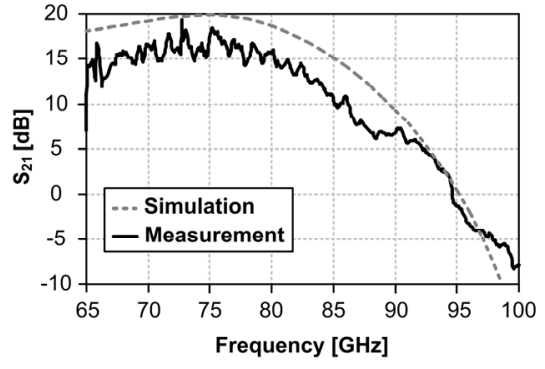

Fig. 7. Small-signal gain $\left(\mathrm{S}_{21}\right)$ of the amplifier simulated and measured between $65 \mathrm{GHz}$ and $100 \mathrm{GHz}$.

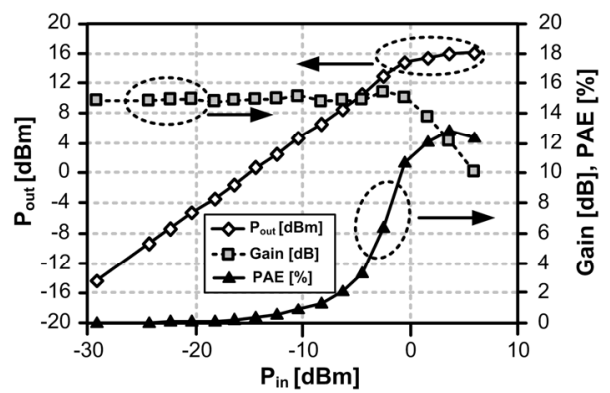

Fig. 8. Measured large-signal parameters of the amplifier at $77 \mathrm{GHz}$.

The large-signal parameters of the amplifier are measured and plotted in Fig. 8. This is measured with a supply voltage of $1.5 \mathrm{~V}$. Amplifier can generate up to $+16 \mathrm{dBm}$, with a compressed gain of $10 \mathrm{~dB}$. A peak PAE of $12.8 \%$ is achieved at peak output power. The output-referred $1 \mathrm{~dB}$ compression point of the amplifier is $14.5 \mathrm{dBm}$. Additional gain and power in the input stages forces the output stage to compress first.

The variation of output saturated power and PAE vs. supply voltage is measured and shown in Fig. 9. Here the amplifier is driven with a constant $+6 \mathrm{dBm}$ input power. Peak output power of $17.5 \mathrm{dBm}$ can be generated with a supply voltage of $1.8 \mathrm{~V}$.

The measured saturated power, gain and PAE of the amplifier vs. frequency are shown in Fig. 10. Peak power and maximum PAE happen exactly at $77 \mathrm{GHz}$, showing the effectiveness of the large-signal power match design methodology outlined in section III.C.

\section{CONCLUSION}

Microstrip tub structure with large isolation has been used to make a $77 \mathrm{GHz}$ power amplifier, fully-integrated in a $0.12 \mu \mathrm{m}$ BiCMOS SiGe process. Amplifier has more than $6 \mathrm{~dB}$ smallsignal gain in a frequency range of $65-95 \mathrm{GHz}$. The measurement of the gain at frequencies lower than $65 \mathrm{GHz}$ is limited by frequency range of the measurement equipment. Large-signal power match has resulted in peak power and PAE occurring at $77 \mathrm{GHz}$. The amplifier operates reliably above the $B V_{C E O}$ range by proper choice of impedance in base and can be used with a supply range of $1 \mathrm{~V}$ to $2.5 \mathrm{~V}$. A comparison of the power amplifier is this work and previous work on mm-wave power amplifiers (mostly in silicon) is summarized in Table I.

\section{ACKNOWLEDGMENT}

The authors would like to thank IBM Microelectronics for fabricating the chip. They also appreciate valuable help from $\mathrm{S}$. Weinreb, A. Babakhani, A. Natarajan, B. Analui, X. Guan, and

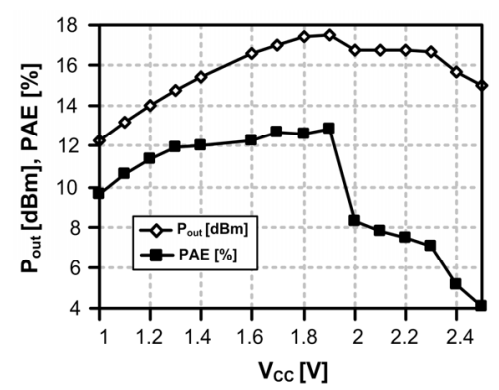

Fig. 9. Measured saturated power and PAE for a supply range of 1-2.5V.

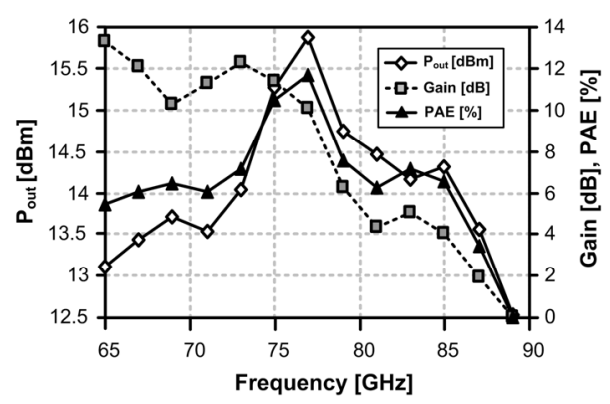

Fig. 10. Measured saturated power, gain and PAE vs. frequency.

E. Afshari. The technical support for CAD tools from Agilent Technologies, Zeland Software Inc. and Ansoft Corp. is also appreciated.

\begin{tabular}{llllcc}
\multicolumn{7}{c}{ Table I. Comparison } \\
\hline Freq. & Device & P out $[\mathrm{dBm}]$ & PAE $_{\max }[\%]$ & Gain [dB] & Ref. \\
\hline $77 \mathrm{GHz}$ & $0.12 \mu \mathrm{m} \mathrm{SiGe}$ & 17.5 & 12.8 & 17 & This Work \\
$77 \mathrm{GHz}$ & $0.12 \mu \mathrm{m} \mathrm{SiGe}$ & 10 & 3.5 & 6.1 & {$[2]$} \\
$60 \mathrm{GHz}$ & $0.12 \mu \mathrm{m} \mathrm{SiGe}$ & 16 & 4.3 & 10.8 & {$[3]$} \\
$90 \mathrm{GHz}$ & $\begin{array}{l}0.1 \mu \mathrm{m} \mathrm{GaAs} \\
\text { pHEMPT }\end{array}$ & 21 & 8 & 19 & {$[9]$} \\
& & & & \\
\hline
\end{tabular}

\section{REFERENCES}

[1] P. Smulders, "Exploiting the 60GHz Band for Local Wireless Multimedia Access: Prospects and Future Directions," IEEE Communications Magazine, vol. 40, no. 1, pp. 140-147, Jan. 2002.

[2] U. R. Pfeiffer, S. K. Reynolds, and B. A. Floyd, "A 77GHz SiGe Power Amplifier for Potential Applications in Automotive Radar Systems," IEEE RFIC Symp. Dig. Papers, pp. 91-94, Jun. 2004.

[3] B. A. Floyd et al., "SiGe Bipolar Transceiver Circuits Operating at 60GHz," IEEE Journal of Solid-State Circuits, vol. 40, no. 1, pp. 156-167, Jan. 2005.

[4] A. Natarajan, A. Komijani, and A. Hajimiri, "A 24GHz Phased-Array Transmitter in $0.18 \mu \mathrm{m}$ CMOS," IIEEE Int. Solid-State Circuits Conf., vol. 48, pp. 212-213, Feb. 2005.

[5] H. Hashemi, X. Guan, A. Komijani, and A. Hajimiri, "A 24GHz SiGe Phased-Array Receiver-LO Phase-Shifting Approach," IEEE Trans. Microwave Theory and Techniques, vol. 53, no. 2, pp. 614-626, Feb. 2005.

[6] J. Grzyb, I. Ruiz, M. Klemm, and G. Troster, "MCM-D Technology for Realization of Low Cost System-on-Package Concept at 60-80GHz," Electrical Performance of Electronic Packaging, pp. 55-58, Oct. 2002.

[7] B. Jagannathan et al., "Self-Aligned SiGe NPN Transistors with $285 \mathrm{GHz}$ $f_{\max }$ and $207 \mathrm{GHz} f_{T}$ in a Manufacturable Technology," IEEE Electron Device Ltrs., vol. 23, no. 5, pp. 258-260, May 2002.

[8] E. O. Johnson, "Physical Limitations on Frequency and Power Parameters of Transistors," IRE International Convention Record, vol. 13, pp. 27-34, Mar. 1965.

[9] K. K. Ng, M. R. Frei, and C. A. King, "'Reevaluation of the $f_{T} B V_{\text {ceo }}$ Limit on Si Bipolar Transistors," IEEE Trans. on Electron Devices, vol. 45, no. 8, pp. 18541855, Aug. 1998.

[10]A. Komijani and A. Hajimiri, "A 24GHz, $+14.5 \mathrm{dBm}$ Fully-Integrated Power Amplifier in $0.18 \mu \mathrm{m}$ CMOS," Proceedings of the IEEE Custom Integrated Circuits Conference, pp. 561-564, Oct. 2004

[11] D.M. Pozar, Microwave Engineering, 2nd ed., John Wiley \& Sons, 1998.

[12] M. Morgan and S. Weinreb, "A W-Band Monolithic Power Amplifier," IEEE Int. Symp. on Microwave Theory and Techniques, vol. 1, pp. 133-136, Jun. 2003. 